\title{
Novel Sequence Variation Affects GPIb $\alpha$ in Post-diarrheal Hemolytic Uremic Syndrome
}

\section{Elena Volokhina ${ }^{1}$, Arjen Jakobi², Rolf Urbanus ${ }^{3}$, Eric Huizinga ${ }^{2}$, Henk Sluiter ${ }^{4}$, Cees Middel ${ }^{5}$, Dineke Westra' ${ }^{1}$, Waander van Heerde ${ }^{5}$, Nicole van de Kar ${ }^{1}$ and Lambertus van den Heuvel ${ }^{1,5,6 *}$}

${ }^{1}$ Department of Pediatric Nephrology, Radboud University Nijmegen Medical Center, The Netherlands

${ }^{2}$ Department of Crystal and Structural Chemistry, Bijvoet Center for Biomolecular Research, Utrecht University, The Netherlands

${ }^{3}$ Department of Clinical Chemistry and Hematology, University Medical Center Utrecht, The Netherlands

${ }^{4}$ Department of Nephrology, Deventer Hospital, The Netherlands

${ }^{5}$ Department of Laboratory, Medicine, Radboud University Nijmegen Medical Centre, The Netherlands

${ }^{6}$ Department of Pediatrics, University Hospitals Leuven, Belgium

\section{Abstract}

Background: Hemolytic uremic syndrome (HUS) is one of the major causes of renal failure in children. In most cases the disease is caused by infection with Shiga toxin- producing Escherichia coli (STEC) and preceded by diarrhea. Only in $15 \%$ of cases STEC infection leads to HUS. Genetic predisposition of a patient to develop HUS after STEC infection might play a role, but very few reports on this subject are available. We describe a novel missense mutation in the GP1BA gene encoding platelet-receptor glycoprotein Iba (GPIba) in a severely affected HUS patient.

Methods: GP1BA was screened by Sanger sequencing. Binding of recombinant GPIba and von Willebrand factor (VWF) fragments was analyzed using surface plasmon resonance (SPR). The hematological studies using patient blood were performed.

Results: The detected heterozygous mutation p.Pro46Leu is located in the proximity to one of the two GPIba-VWF binding sites. SPR experiments show that the p.Pro46Leu leads to a small increase in GPIba-VWF binding $(p<0.001)$. The hemostatic parameters of patient blood after recovery from HUS show normal values.

Conclusions: The described mutation affects GPIba interaction with VWF in a mild gain-of-function manner and might have contributed to a prothrombotic state in the patient and to development of HUS.

Keywords: DNA change; GPIba; Hemolytic uremic syndrome; Platelets; Von Willebrand factor

\section{Introduction}

The Hemolytic Uremic Syndrome (HUS) is one of the major causes of renal failure in children. It is characterized by hemolytic anemia, thrombocytopenia and acute renal failure [1]. More than $90 \%$ of cases (typical HUS) are preceded by watery or bloody diarrhea and are caused by an infection with Shiga toxin-producing Escherichia coli (STEC), which can be ingested with improperly cooked meat and contaminated vegetables and water [2]. The majority of typical HUS cases affects children and is associated with infection by O157:H7 STEC serotype. The STEC infection leads to typical HUS in approximately $15 \%$ of cases [3]. Interestingly, the outbreak in Germany in 2011 was caused by an unusual O104:H4 strain, which mostly affected adults [4]. In the intestine, HUS-causing STEC strains produce Shiga toxins, belonging to type 1 and/or type $2[5,6]$. The toxins pass the intestinal wall and enter the circulation; they are recognized by the glycolipid receptor globotriaosylceramide (Gb3) on the surface of various cell types. The exact mechanism of how exposure to Shiga toxins leads to HUS and the reason why glomerular endothelium of the kidney is most strongly affected in HUS is poorly understood. Shiga toxins are known to inhibit protein synthesis and are involved in the induction of apoptosis [7]. Complement activation by Shiga toxin on endothelial cells was shown in vitro, in a murine HUS model [8] and on platelet-leukocyte complexes and microparticles of HUS patients [9]. In vitro studies showed that Shiga toxin 2 binds complement factor $\mathrm{H}(\mathrm{CFH})$, thereby reducing $\mathrm{CFH}$ complement inhibiting activity at the cell surface [10]. Interestingly, $85 \%$ of individuals that are infected with STEC will not progress to HUS.

In atypical HUS (aHUS), which is not preceded by the STEC infection, $50-60 \%$ of patients carry predisposing changes in complement genes encoding $\mathrm{CFH}$, complement factor I (CFI), membrane cofactor protein (MCP), C3, complement factor B (CFB) and thrombomodulin and/or autoantibodies to CFH [11]. Similar to aHUS, genetic predisposition might be important in typical HUS. A single study reported significantly different frequencies of the three missense single nucleotide polymorphisms (SNPs) in the group of typical HUS patients, compared to healthy controls [12]. In the GP1BA gene, which encodes platelet-receptor glycoprotein Iba (GPIba), a higher frequency of the (human platelet antigen 2) HPA2 SNP was strongly associated with typical HUS. Furthermore, significantly different frequencies among HUS patients were reported for the two SNPs in genes encoding adipocyte-derived leucine aminopeptidase and factor V. Another report described a single mutation in MCP that was found in a typical HUS patient and also in patients with aHUS, HELLP (hemolysis, elevated liver enzymes, and low platelets) syndrome, and glomerulonephritis with C3 deposits [13]. Gaining further insight into the genetic background in typical HUS is important as it could improve our understanding of pathogenesis of the disease and possibly provide new possibilities for the treatment of patients. Here we describe a novel sequence variation in the GP1BA gene found

*Corresponding author: van den Heuvel L.P, Department of Pediatric Nephrology (774) Radboud University Nijmegen Medical Center P.O. Box 9101, 6500 HB Nijmegen, The Netherlands, Tel: +31-24-3617983; Fax: +31-24-3618900; E-mail: b.vandenheuvel@cukz.umcn.nl

Received February 04, 2014; Accepted March 22, 2014; Published March 27 2014

Citation: Volokhina E, Jakobi A, Urbanus R, Huizinga E, Sluiter H, et al. (2014) Novel Sequence Variation Affects GPIba in Post-diarrheal Hemolytic Uremic Syndrome. J Nephrol Therapeutic S11: 007. doi:10.4172/2161-0959.S11-007

Copyright: ( 2014 Volokhina E, et al. This is an open-access article distributed under the terms of the Creative Commons Attribution License, which permits unrestricted use, distribution, and reproduction in any medium, provided the original author and source are credited. 
in a post-diarrheal (typical) HUS patient and provide evidence for its functional consequence.

\section{Methods}

\section{Genetic analysis}

Genomic DNA was isolated from peripheral blood leukocytes as described before [14]. Coding sequences of GP1BA, C3, CFH, CFI, $M C P$ and $C F B$ were amplified by PCR; primer sequences are available upon request. The amplimers were subjected to double-stranded DNA sequence analysis on an ABI $3130 x l$ Genetic Analyzer (Applied Biosystems). Presence of the novel genetic change was analyzed among 192 ethnically-matched control subjects. To this end, commercially available Human Random Control DNA panels were obtained (SigmaAldrich).

\section{Autoantibodies to CFH}

Patient serum was tested for the presence of autoantibodies to $\mathrm{CFH}$ by means of ELISA as described before [15]. A test result was considered positive if obtained extinction value was more than twice the standard deviation above the mean value calculated from 16 controls. The assay was repeated three times; a positive control sample was taken in every measurement.

\section{Hematological analyses}

Ristocetin induced platelet agglutination was analyzed in Platelet Rich Plasma using 0.5, 0.6, 0.7, 0.8, 0.9 and $1.52 \mathrm{mg} / \mathrm{ml}$ of ristocetin. Agglutination was analyzed in a Chronolog Aggregometer. All other hematological analyses were performed according to the standard operational protocols used at the RUNMC, which are available upon request.

\section{Recombinant protein production}

GPIba residues (1-290) containing p.Asn37Gln and p.Asn175Gln mutations to remove $\mathrm{N}$-glycosylation sites, preceded by a cystatin signal peptide and fused to a C-terminal Arg-(His)6 sequence, were cloned into a modified pUPE expression vector. The p.Pro46Leu sequence variation and p.Met255Val, a platelet-type von Willebrand disease mutation were introduced using the Quick Change method. Proteins were expressed in HEK-293-EBNA1-S cells and purified as previously described [16]. Fully sulfated protein was concentrated to $\sim 1 \mathrm{mg} / \mathrm{ml}$ in the gel filtration buffer $(50 \mathrm{mM} \mathrm{NaCl}, 20 \mathrm{mM}$ Tris/HCl pH 8.0).

Wild type von Willebrand factor A1 domain (VWF-A1) and VWF-A1 carrying the p.Arg1306Gln type $2 \mathrm{~B}$ von Willebrand disease mutation (both residues 1,261-1,468) were expressed and purified as described [17] and subsequently concentrated to $\sim 5 \mathrm{mg} / \mathrm{mL}$ in $100 \mathrm{mM}$ $\mathrm{NaCl}, 15$ mM HEPES pH 7.5.

\section{Surface plasmon resonance experiments}

Surface plasmon resonance (SPR) binding studies were performed on a Biacore T100 (GE Healthcare). Approximately 9500 response units (RU) of GPIba monoclonal antibody 2D4 were immobilized on a series S CM5 sensor chips by amine coupling as instructed by the supplier. A control channel was activated and blocked by using the amine coupling reagents in the absence of protein. Proteins were dialyzed to standard SPR buffer $(150 \mathrm{mM} \mathrm{NaCl}, 0.005 \%$ (v/v) Tween-20 and $25 \mathrm{mM}$ HEPES $\mathrm{pH}$ 7.4) and analyzed at $25^{\circ} \mathrm{C}$. GPIb $\alpha$ variants (wild type, p.Met255Val and p.Pro46Leu, each $150 \mathrm{nM}$ ) were injected for 60 seconds at $10 \mu \mathrm{l} / \mathrm{min}$, followed by a 60 seconds association phase of wild type VWF-A1 (0-4000 nM) or p.Arg1306Gln VWF-A1 (0-1000 nM) and a $120 \mathrm{sec}$ dissociation phase at $30 \mu \mathrm{l} / \mathrm{min}$. Proteins were injected until binding equilibrium was reached. Between runs, the sensor chip was regenerated by injections of $50 \mathrm{mM}$ triethylamine, $10 \mathrm{mM}$ sodium formate $\mathrm{pH} 2.0$ containing $150 \mathrm{mM} \mathrm{NaCl}$ and another injection of 50 $\mathrm{mM}$ triethylamine. Each interaction was analyzed at seven different VWF-A1 concentrations. Data evaluation was performed with BIAcore T100 evaluation software. Affinity constants were derived by steady state analysis. Kinetics analysis was not possible due to the instrument limitations.

\section{Results}

\section{Case report}

The female patient is the youngest child in a family with two children of healthy parents. In 1977, at the age of 6.5 years the patient developed HUS. The initial symptoms included diarrhea, extreme paleness, fatigue and vomiting. The laboratory findings demonstrated anemia, with presence of fragmentocytes, and high creatinine and urea serum values. The patient was started on peritoneal dialysis, which was performed six times; heparinization was used, but was stopped after an intestinal bleeding episode. A Scribner shunt was placed in the leg for hemodialysis. Within the two months after first symptoms the patient suffered two cerebral accidents, possibly due to hypertensive encephalopathy. The patient developed hypertension, which was stabilized by medication (Nepresol, Inderal, Catapresan).

At the age of nine years, the patient received a first cadaver kidney transplant. At the age of 17, the kidney graft was removed due to chronic rejection and the patient was referred for hemodialysis for three years. After that, at the age of 20 , she received a second cadaver kidney transplant, which was removed 14 years later due to chronic rejection, during this period the patient also suffered from cytomegalovirus infection. Two years later the patient received a kidney graft from a living donor via cross-over transplantation program, where the mother donated a kidney to another patient. At the moment of the study, the patient had a stable kidney graft function for five years. The function of the remaining kidney of the mother is also normal. There was no HUS recurrence reported in any of the grafts.

As the patient in question was diagnosed with HUS in 1977, presence of the STEC infection was not investigated. The STEC involvement in HUS was only demonstrated in 1983 [18] therefore it was not possible to test this patient for the presence of STEC in the acute phase of the disease. Nevertheless, presence of diarrhea, the young age of the patient, and lack of recurrence in kidney grafts support the possibility that the patient had suffered from the STEC-associated typical HUS, rather than from the atypical form of HUS.

\section{Novel GP1BA alteration found in HUS patient}

The objective of this study was to investigate whether this severely affected patient carries genetic predisposition to the development of HUS. No DNA alterations were found in genes encoding aHUSpredisposing factors $\mathrm{CFH}, \mathrm{CFI}, \mathrm{MCP}, \mathrm{C} 3, \mathrm{CFB}$ and thrombomodulin. Moreover, autoantibodies to $\mathrm{CFH}$ were not detected in the patient. Previously, a strong association between typical HUS and GP1BA SNP was described [12]. We, therefore, screened the coding sequence of GP1BA. A single novel heterozygous missense sequence variation c.137C $>\mathrm{T}$ (p.Pro46Leu) was identified (Figure 1a). This novel sequence variation was also found in the DNA of patient's brother (16 month older than the patient) and her father that were both not affected by HUS. The patient did not carry the HPA2 polymorphism. The DNA change was neither described previously, nor found by us in 192 
Citation: Volokhina E, Jakobi A, Urbanus R, Huizinga E, Sluiter H, et al. (2014) Novel Sequence Variation Affects GPIba in Post-diarrheal Hemolytic Uremic Syndrome. J Nephrol Therapeutic S11: 007. doi:10.4172/2161-0959.S11-007

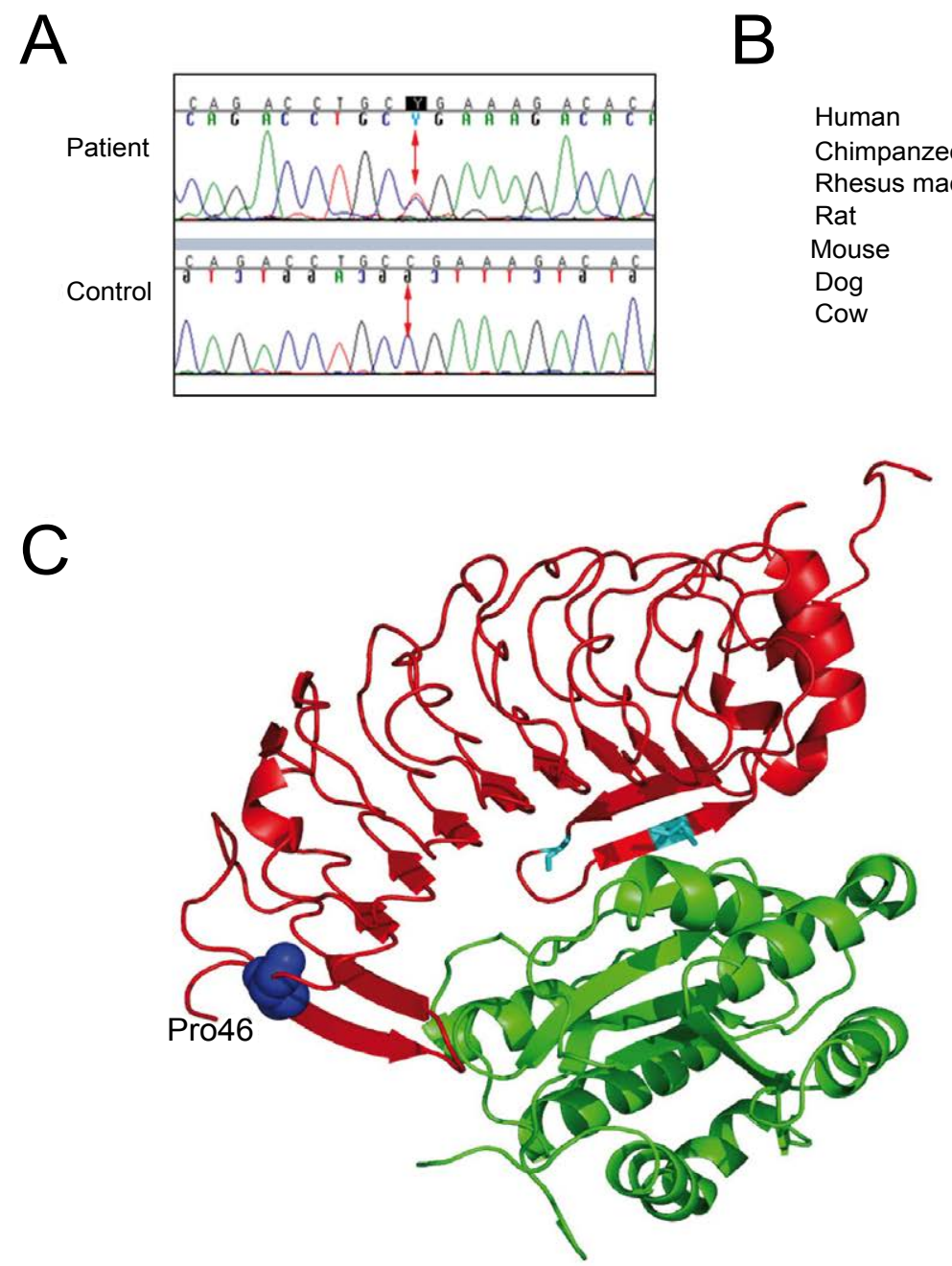

Figure 1: The novel missense sequence variation in GPIba affects a conserved amino acid in the HUS patient. a. Sequencing chromatograms of the HUS patient with c.137C > T (top) and of a control individual without the change (bottom). The position of the altered nucleotide is indicated with an arrow. b. Sequence alignment of corresponding regions of GPIba homologues from various species: human (NP 000164.5); chimpanzee (XP 523557.3); rhesus macaque (ACJ38405.1); rat (NP_001103124.1); mouse (NP_034456.2); dog (NP_001003083.1); cow (XP_002695841.1), the Pro46 position is indicated. c. Structure of the N-terminal part of GPIba (red) in complex with VWF-A1 domain (green) (protein data bank identification code: $1 \mathrm{M} 10)$. The Pro46, affected by the novel genetic change, is indicated with dark blue spheres, Met255 and Gly249, mutated in platelet-type von Willebrand disease are shown in light blue.

healthy control individuals. The variation affected an evolutionary conserved amino acid residue (Figure $1 \mathrm{~b}$ ) and was suggested to alter the GPIba protein function by mutation analysis programs SIFT (score 0.00, http://sift.jcvi.org/) and PolyPhen-2 (possibly damaging, http:// genetics.bwh.harvard.edu/pph2/). Therefore, the found DNA change was likely to affect GPIba protein function and might have facilitated the development of HUS in the patient.

\section{Novel alteration enhances GPIba interaction with VWF}

The identified novel DNA change affected an amino acid residue in the proximity of one of the two interaction sites between GPIba N-terminal part and the VWF-A1 domain [16] (Figure 1c). Since formation of microthrombi in capillaries is a prominent feature of HUS, we hypothesized that the p.Pro46Leu change is prothrombotic. The missense alteration might have strengthened the GPIba and VWF interaction and in this way contributed to the formation of microthrombi in HUS. We, therefore, investigated the impact of the novel sequence variation on VWF binding using purified recombinant proteins. The binding of the GPIba N-terminal fragment to the VWF-A1 domain was measured by SPR. The p.Pro46Leu change in GPIba led to a small increase in steady state affinity for the VWF-A1 domain $(\mathrm{p}<0.001)$ (Table 1). Approximately $10 \%$ decrease in dissociation constant $(\mathrm{Kd})$ was observed for the interaction with wild type VWF-A1 as well as with a variant carrying the p.Arg1306Gln mutation that stabilizes the interaction and is associated with type $2 \mathrm{~B}$ von Willebrand disease [16]. The increase in affinity for the p.Pro46Leu variant was much smaller than for the gain-of-function p.Met255Val change in GPIba, which is associated with platelet-type von Willebrand disease and strengthens the interaction more than 30 fold (Table 1).

\section{Hematological findings}

The SPR experiments suggested a mild increase in GPIba affinity to VWF. We further performed extensive hematological studies to determine whether hemostasis is affected in the patient. The routine hematological analysis revealed no abnormalities, except for elevated factor-VIII activity (Table 2). In particular, we were interested in 


\begin{tabular}{|c|c|c|}
\hline Immobilized ligand & Analyte & KD (nM) \\
\hline wta GPIba & wt VWF-A1 & $690 \pm 3 \mathrm{nM}$ \\
\hline & p.Arg1306GIn VWF -A1 & $69 \pm 0.2 \mathrm{nM}$ \\
\hline p.Pro46Leu GPIba & wt VWF-A1 & $630 \pm 2 \mathrm{nM}$ \\
\hline & p.Arg1306GIn VWF -A1 & $61 \pm 0.4 \mathrm{nM}$ \\
\hline p.Met255Val GPIba & wt VWF-A1 & $18 \pm 0.09 \mathrm{nM}$ \\
\hline & p.Arg1306Gln VWF -A1 & $6 \pm 0.05 \mathrm{nM}$ \\
\hline
\end{tabular}

awt stands for wild type

Table 1: Summary of the SPR steady state analysis.

\begin{tabular}{|c|c|c|}
\hline Assay & Patient value & Normal range \\
\hline Hemoglobin & $7.7 \mathrm{mmol} / \mathrm{l}$ & 7.3-9.7 mmol/l \\
\hline Hematocrit & 0.38 & $0.34-0.46$ \\
\hline Thrombocytes & $243 \times 109 / 1$ & $120 \times 109-350 \times 109 / 1$ \\
\hline Mean platelet volume & $7.6 \mathrm{fl}$ & $7.5-11.5 \mathrm{fl}$ \\
\hline Ristocetin cofactor activity (VWF) & $165 \%$ & $50-185 \%$ \\
\hline VWF multimers & Normal & $\mathrm{NAa}$ \\
\hline Collagen binding assay & $144 \%$ & $64-155 \%$ \\
\hline Factor-VIII activity & $180 \%$ & $60-150 \%$ \\
\hline ADP aggregation & Normal & $\mathrm{NA}^{\mathrm{a}}$ \\
\hline Collagen aggregation & Normal & $N A^{a}$ \\
\hline Arachidonic acid aggregation & Normal & $\mathrm{NA}^{\mathrm{a}}$ \\
\hline PFA (collagen-epinefrin) & $97 \mathrm{sec}$ & $<170 \mathrm{sec}$ \\
\hline PFA (collagen-ADP) & $74 \mathrm{sec}$ & $<120 \mathrm{sec}$ \\
\hline Ristocetin agglutination $^{b}$ & Normal & $N A^{a}$ \\
\hline GP53 (CD63) & Normal & $N A^{a}$ \\
\hline GPIIbllla (PAC-1) & Normal & $\mathrm{NA}^{\mathrm{a}}$ \\
\hline P-selectin (CD62p) & Normal & $N A^{a}$ \\
\hline
\end{tabular}

aNA stands for not applicable, no reference values are known, the patient sample was compared to the normal control samples.

${ }^{\mathrm{b}}$ The platelet aggregation was measured using platelet rich plasma in the presence of $0.5,0.6,0.7,0.8,0.9$ and $1.52 \mathrm{mg} / \mathrm{ml}$ of ristocetin.

Table 2: Hematological analysis of patient carrying GPIba sequence variation.

features that could indicate abnormal platelet function: mean platelet volume, associated with higher platelet activity, aggregation and prothrombotic potential; platelet activation markers (CD63, PAC-1, $\mathrm{CD} 62 \mathrm{p}$ ) and ristocetin-induced platelet agglutination. The ristocetininduced platelet agglutination was of particular interest, because ristocetin triggers platelet agglutination by inducing interaction between GPIba and VWF [19]. Platelet agglutination was measured using platelet- rich plasma in the presence of $0.5,0.6,0.7,0.8,0.9$ and $1.52 \mathrm{mg} / \mathrm{ml}$ of ristocetin. Agglutination in the patient sample was induced at $0.7 \mathrm{mg} / \mathrm{ml}$ ristocetin, which was similar to the samples from healthy controls.

\section{Discussion}

In this work we describe a novel genetic change in the GP1BA gene in a post- diarrheal HUS patient and its functional characterization. Since this genetic change affects an amino acid in the proximity to one of the VWF-A1 binding sites, we analyzed GPIba binding to this major ligand. In our SPR experiments using recombinant proteins the sequence variation was associated with a mild increase in affinity between GPIb $\alpha$ and VWF-A1 domain. Previously, in vitro experiments using human umbilical vein endothelial cells and glomerular microvascular endothelial cells have shown that Shiga toxin triggers secretion of unusually large VWF multimers (ULVWF), which have increased affinity to platelets. Such multimers were not present in the patient's blood at the time of the study (Table 2), Since the blood that was used in experiments was collected long after the patient had suffered from HUS. Furthermore, presence of the Shiga toxin delays VWF multimer cleavage by ADAMTS13, promoting platelet adhesion [20]. In this particular HUS patient platelet adhesion might have been further enhanced by the novel genetic change, contributing to the formation of thrombi in microcirculation of the patient and to development of HUS.

Although in this study we focused on the GPIba-VWF interface, p.Pro46Leu might also have an impact on GPIba binding with other ligands, such as thrombin, $\mathrm{P}$ - selectin, protein $\mathrm{C}$ and clotting factors XI and XII [21]. P-selectin might be particularly interesting, since its production is upregulated by Shiga toxin [22]. Lately this upregulation was linked to complement activation and thrombus formation induced by Shiga toxin in vitro and in murine model [8].

Recent study demonstrated that Listeria monocytogenes binds to platelets in the blood in a manner dependent on GPIb and C3 in mice [23]. This finding might implicate GPIb in complement biology, which might also be relevant in HUS.

The brother and father of the patient carry the same genetic alteration in GP1BA but were never affected by HUS. In particular, the absence of HUS in the brother is interesting because this disease mostly affects children [2]. However, it is possible that the brother was not exposed to STEC.

Various mutations in GPIba were previously described in relation to human disease. Gain-of-function mutations that increase GPIbaVWF affinity cause platelet- type von Willebrand disease, the wellknown mutations are p.Met255Val and p.Gly249Val (often in literature referred to as p.Met239Val and p.Gly233Val, when not counting the signal peptide) (Figure 1c). Effect of p.Pro46Leu, measured by us using SPR, is much less than that of p.Met255Val (Table 1). This is not unexpected, since our patient does not have platelet-type von Willebrand disease.

Our results indicate that genetic changes that affect GPIba interactions might be relevant in typical HUS. Furthermore, it is possible that gain-of-function GP1BA sequence variations might be relevant in the etiology of thrombotic thrombocytopenic purpura, a thrombotic microangiopathy, where decreased ADAMTS13 activity leads to inefficient cleavage of the highly potent ULVWF multimers [24]. Similar deficiencies might be involved in both typical and atypical HUS, such as the MCP mutation mentioned earlier [13]. Therefore, involvement of GP1BA deficiencies in atypical HUS is also possible. We screened this gene in our cohort of 64 aHUS patients [25]. No novel genetic changes or SNP frequencies different than reported for European population (http://www.ncbi.nlm.nih.gov/pubmed/) were observed (data not shown), however due to the relatively small number of patients in our cohort the involvement of GP1BA changes in aHUS pathology still cannot be excluded.

\section{Acknowledgements}

We would like to thank B. van Haren and J.C.M. Vos for practical assistance; B. Verbruggen for precious advice; patients and their parents for participation in this study. We thank H. Deckmyn, KU Leuven, for providing 2D4 antibody. E.B. Volokhina and D. Westra were supported by RUNMC and Dutch Kidney Foundation A.J. Jakobi was supported by an ECHO grant from the Netherlands Foundation of Scientific Research and R.T. Urbanus was supported by the Dutch Heart Foundation.

\section{References}

1. GASSER C, GAUTIER E, STECK A, SIEBENMANN RE, OECHSLIN R (1955) [Hemolytic-uremic syndrome: bilateral necrosis of the renal cortex in acute acquired hemolytic anemia]. Schweiz Med Wochenschr 85: 905-909. 
Citation: Volokhina E, Jakobi A, Urbanus R, Huizinga E, Sluiter H, et al. (2014) Novel Sequence Variation Affects GPIba in Post-diarrheal Hemolytic Uremic Syndrome. J Nephrol Therapeutic S11: 007. doi:10.4172/2161-0959.S11-007

Page 5 of 5

2. Noris M, Remuzzi G (2005) Hemolytic uremic syndrome. J Am Soc Nephrol 16: $1035-1050$

3. Tarr PI, Gordon CA, Chandler WL (2005) Shiga-toxin-producing Escherichia coli and haemolytic uraemic syndrome. Lancet 365: 1073-1086.

4. Frank C, Werber D, Cramer JP, Askar M, Faber M, et al. (2011) Epidemic profile of Shiga-toxin-producing Escherichia coli O104:H4 outbreak in Germany. N Engl J Med 365: 1771-1780.

5. Nakao H, Takeda T (2000) Escherichia coli Shiga toxin. J Nat Toxins 9: 299313.

6. Strockbine NA, Marques LR, Newland JW, Smith HW, Holmes RK, et al. (1986) Two toxin-converting phages from Escherichia coli 0157:H7 strain 933 encode antigenically distinct toxins with similar biologic activities. Infect Immun 53: 135140

7. Johannes L, Römer W (2010) Shiga toxins--from cell biology to biomedical applications. Nat Rev Microbiol 8: 105-116.

8. Morigi M, Galbusera M, Gastoldi S, Locatelli M, Buelli S, et al. (2011) Alternative pathway activation of complement by Shiga toxin promotes exuberant $\mathrm{C} 3 \mathrm{a}$ formation that triggers microvascular thrombosis. J Immunol 187: 172-180.

9. Ståhl AL, Sartz L, Karpman D (2011) Complement activation on plateletleukocyte complexes and microparticles in enterohemorrhagic Escherichia coli-induced hemolytic uremic syndrome. Blood 117: 5503-5513.

10. Orth D, Khan AB, Naim A, Grif K, Brockmeyer J, et al. (2009) Shiga toxin activates complement and binds factor $\mathrm{H}$ : evidence for an active role of complement in hemolytic uremic syndrome. J Immunol 182: 6394-6400.

11. Noris M, Remuzzi G (2009) Atypical hemolytic-uremic syndrome. N Engl J Med 361: 1676-1687

12. Taranta A, Gianviti A, Palma A, De Luca V, Mannucci L, et al. (2009) Genetic risk factors in typical haemolytic uraemic syndrome. Nephrol Dial Transplan 24: 1851-1857.

13. Fang CJ, Fremeaux-Bacchi V, Liszewski MK, Pianetti G, Noris M, et al. (2008) Membrane cofactor protein mutations in atypical hemolytic uremic syndrome (aHUS), fatal Stx-HUS, C3 glomerulonephritis, and the HELLP syndrome. Blood 111: 624-632.

14. Miller SA, Dykes DD, Polesky HF (1988) A simple salting out procedure for extracting DNA from human nucleated cells. Nucleic Acids Res 16: 1215.
15. Józsi M, Strobel S, Dahse HM, Liu WS, Hoyer PF, et al. (2007) Anti factor H autoantibodies block $\mathrm{C}$-terminal recognition function of factor $\mathrm{H}$ in hemolytic uremic syndrome. Blood 110: 1516-1518.

16. Huizinga EG, Tsuji S, Romijn RA, Schiphorst ME, de Groot PG, et al. (2002) Structures of glycoprotein Ibalpha and its complex with von Willebrand facto A1 domain. Science 297: 1176-1179.

17. Jakobi AJ, Mashaghi A, Tans SJ, Huizinga EG (2011) Calcium modulates force sensing by the von Willebrand factor A2 domain. Nat Commun 2: 385.

18. Karmali MA, Steele BT, Petric M, Lim C (1983) Sporadic cases of haemolyticuraemic syndrome associated with faecal cytotoxin and cytotoxin-producing Escherichia coli in stools. Lancet 1: 619-620.

19. Shen Y, Romo GM, Dong JF, Schade A, McIntire LV, et al. (2000) Requirement of leucine-rich repeats of glycoprotein (GP) Ibalpha for shear-dependent and static binding of von Willebrand factor to the platelet membrane GP lb-IX-V complex. Blood 95: 903-910.

20. Nolasco LH, Turner NA, Bernardo A, Tao Z, Cleary TG, et al. (2005) Hemolytic uremic syndrome-associated Shiga toxins promote endothelial-cell secretion and impair ADAMTS13 cleavage of unusually large von Willebrand facto multimers. Blood 106: 4199-4209.

21. Clemetson KJ, Clemetson JM (2008) Platelet GPIb complex as a target for antithrombotic drug development. Thromb Haemost 99: 473-479.

22. Morigi M, Galbusera M, Binda E, Imberti B, Gastoldi S, et al. (2001) Verotoxin-1 induced up-regulation of adhesive molecules renders microvascular endothelial cells thrombogenic at high shear stress. Blood 98: 1828-1835.

23. Verschoor A, Neuenhahn M, Navarini AA, Graef P, Plaumann A, et al. (2011) A platelet-mediated system for shuttling blood-borne bacteria to CD8Ît+ dendritic cells depends on glycoprotein GPIb and complement C3. Nat Immunol 12 1194-1201.

24. Moake JL (2004) von Willebrand factor, ADAMTS-13, and thrombotic thrombocytopenic purpura. Semin Hematol 41: 4-14.

25. Westra D, Volokhina E, van der Heijden E, Vos A, Huigen M, et al. (2010) Genetic disorders in complement (regulating) genes in patients with atypical haemolytic uraemic syndrome (aHUS). Nephrol Dial Transplant 25: 2195-2202.
This article was originally published in a special issue, Pediatric Nephrology handled by Editor(s). Moazzam Hossain, Bangabandhu Sheikh Mujib Medica University, Bangladesh 